\title{
Realization of TDOA Estimation Architectures
}

\author{
Herschel H. Loomis, Jr. and Raymond F. Bernstein, Jr. \\ Department of Electrical and Computer Engineering \\ Naval Postgraduate School, Monterey, California 93943
}

\begin{abstract}
An architecture for the computation of time difference of arrival (TDOA) estimates is proposed. The structure of the various principal components is developed and a complexity analysis is presented. Realization using pipeline FFT and vector processing chips is considered. Conclusions are reached concerning the prefered spectral correlation and TDOA computing algorithms.
\end{abstract}

\section{Introduction}

Spectral correlation techniques have been shown to be useful in:

- signal detection in the presence of noise [1]

- signal characterization and parameterization [1];

- signal source location [2], [3];

Signal detection and characterization relies on the computation of a smoothed cyclic periodogram:

$$
S_{x_{T}}^{\alpha}(t, f)=\frac{1}{T} X_{T}(t, f+\alpha / 2) X_{T}^{*}(t, f-\alpha / 2),
$$

where,

$$
X_{T}(t, f)=\int_{t-T / 2}^{t+T / 2} x(u) e^{-i 2 \pi f u} d u
$$

There are basically two smoothing approaches, frequency smoothing, and time smoothing. [4] [5]

- Frequency Smoothing $(T=\Delta t)$ :

$$
\begin{aligned}
S_{x_{\Delta t}}^{\alpha}(t, f)_{\Delta f} & =\frac{1}{\Delta f} \int_{f-\Delta f / 2}^{f+\Delta f / 2} S_{x_{\Delta t}}^{\alpha}(t, v) d v, \\
\hat{S}_{x}^{\alpha}(f) & =\lim _{\Delta f \rightarrow 0} \lim _{\Delta t \rightarrow \infty} S_{x_{\Delta t}}^{\alpha}(t, f)_{\Delta f}
\end{aligned}
$$

- Time Smoothing $(T=1 / \Delta f)$ :

$$
\begin{gathered}
S_{x_{1 / \Delta f}}^{\alpha}(t, f)_{\Delta t}=\frac{1}{\Delta t} \int_{t-\Delta t / 2}^{t+\Delta t / 2} S_{x_{1 / \Delta f}}^{\alpha}(t, \tau) d \tau \\
\hat{S}_{x}^{\alpha}(f)=\lim _{\Delta f \rightarrow 0} \lim _{\Delta t \rightarrow \infty} S_{x_{1 / \Delta f}}^{\alpha}(t, f)_{\Delta t}
\end{gathered}
$$

In practice, the limits in (3) and (5) cannot be approached. Instead, we let $\Delta f \Delta t$ get large.

The time smoothed and frequency smoothed cyclic periodograms will be called Spectral Correlation Functions (SCF) in the rest of this paper because it approaches in the limit the limit spectral correlation function, $\hat{S}_{x}^{\alpha}$. Realizations of SCFs are considered in detail in [4]. Machines which realize the SCF are called Spectral Correlation Analyzers (SCA).

The previous has dealt with what is termed the Auto Spectral Correlation function, $\hat{S}_{x}^{\alpha}(f)$. The Cross Spectral Correlation Function $\hat{S}_{x y}^{\alpha}(f)$ can be computed in a similar fashion to (3) and (5) from the Cross Cyclic Periodogram,

$$
S_{x y_{T}}^{\alpha}(t, f)=\frac{1}{T} X_{T}(t, f+\alpha / 2) Y_{T}^{*}(t, f-\alpha / 2),
$$

An important result was developed in [4] from work originally reported in [3] is that the output signal-tonoise ratio of the SCA is proportional to $\Delta f \Delta t$ in (2) and (4).

The input SNR is defined as the average signal power divided by the average noise power within half the null-to-null bandwidth of the BPSK signal;

$$
\rho_{\text {in }}=\frac{P_{s} T_{b}}{2 N_{0}} .
$$

Computing the SCF for the carrier feature at $\alpha=2 f_{0}$, for cases where $\Delta f=1 / T_{b}$, Brown has shown in [4] that,

$$
\rho_{\text {out }}=0.3 \rho_{\text {in }}^{2} \frac{\Delta t}{T_{b}} .
$$

For $\Delta f$ small compared to $2 / T_{b}$,

$$
\rho_{\text {out }}=\frac{1}{2} \rho_{\text {in }}^{2} \Delta t \Delta f \quad \Delta f<\frac{.5}{T_{b}} .
$$

Similar results can be derived for other features of BPSK and for other PSK modulations.

The SCF can be used to:

- detect cyclostationary signals in the presence of noise, and interference, provided $\Delta f \Delta t$ is suffciently large, 
- measure parameters of signals of interest, such as carrier frequency, $f_{0}$, and data frequency, $f_{b}$, of PSK signals,

- characterize signals into modulation type BPSK, QPSK, MSK, SQPSK [1].

Source location. Once a signal of interest is discovered, then, if two physically separated receivers are available, the time-difference-of-arrival (TDOA) of the two waveforms at the receivers can be compossible emitter positions called an isochron. Known motion of the receivers and/or of the emitter can then give rise to multiple, independent TDOAs, resulting in intersecting isochrons and hence a position determination.

Gardner and Chen have developed cyclic techniques for computing TDOA of a cyclostationary signal received in two locations [2], [3]. These techniques make use of the Cross SCF (CSCF), presented in [8]. Two principal techniques are the Spectral Correlation Ratio (SPECCOR) and Spectral Coherence Alignment (SPECCOA) methods. They are developed and analyzed in detail in [2] and [3].

\section{- SPECCOA}

$$
\begin{gathered}
\hat{\Delta} \sim \max _{\tau} \operatorname{Re} \\
\left\{\int_{-f_{0} / 2}^{f_{s} / 2}\left[S_{x y_{\Delta t}}^{\alpha_{0}}(f)_{\Delta f_{c}}\right]\left[S_{x_{1 / \Delta t}}^{\alpha_{0}}(f)_{\Delta t}^{*}\right] e^{i 2 \pi f \tau} d \tau e^{i \pi \alpha \tau}\right\}
\end{gathered}
$$

\section{- SPECCOR}

$$
\hat{\Delta} \sim \max _{\tau}\left|\int_{B W} \frac{S_{x y_{\Delta t}}^{\alpha_{0}}(f)_{\Delta f_{c}}}{S_{x_{1 / \Delta f}}^{\alpha_{0}}(f)_{\Delta t}} e^{i 2 \pi f \tau} d \tau\right|
$$

In general, the smoothing $\Delta f$ for the cross spectrum $\Delta f_{c}$, is less than $\Delta f$ for the auto spectrum.

Chen and Gardner conclude in [2] that SPECCOA produces a better estimate of the TDOA. In this paper, we analyze the computation of TDOA by these methods.

\section{Hardware Considerations}

Near-real-time Computations. Our objective is to consider ways to implement SCA s in near real time. As an aid to characterizing the closeness to real time computation, we define the Real Time Factor, $F_{T}$ as

$$
F_{T}=\frac{\text { Computation Time }}{\text { Collect Time }} .
$$

We will find it useful to estimate the complexity or number of hardware units $p_{h u}$, needed to operate at a particular, $F_{T}$. This is simply the number of operations performed by that unit, $C_{u}$, divided by the total number of clock periods available to do the operations, $F_{T} \cdot \Delta t$, where $\Delta t$ is the total number of samples processed. Thus,

$$
p_{h u}=\frac{C_{u}}{F_{T} \Delta t}=\frac{C_{u}}{F_{T} N} .
$$

We will use the Complexity Product, $p_{h u} \cdot F_{T}$, as a measure of the hardware complexity of a particular architecture in our subsequent analysis of different schemes for computing the spectral correlation function.

If an algorithm requires $C$ operations to calculate the desired function, and we have $F_{T} \cdot N$ samples in which to do the calculation, the complexity product for rate-r operators will be:

$$
p_{1} \cdot F_{T}=\frac{C}{r \cdot N} .
$$

If we use a rate- $\frac{1}{R}$ operator, it will require $R$ times as many hardware units, so

$$
p_{2} \cdot F_{T}=\frac{R \cdot C_{b}}{N}
$$

FFT Realizations. A fundamental building block of any SCA is the N-point FFT. The fundamental building block of the FFT, in turn, is the decimation in time complex butterfly (abbreviated CBF), which is presented in a number of basic digital signal processing references such as Oppenheim and Schafer [4]. In general, the radix- $R$ butterfly can be constructed which accepts the $R$ input complex numbers in $R$ clock pulse periods and produces, in a pipeline fashion, the $R$ complex output numbers. We emphasize that our pipeline CBF operates at the $1 / R$ rate, that is, the unit produces one CBF calculation of $R$ complex values per $R$ sample periods.

However, our input and output data memories will be each operating at one operand per clock period, furnishing the $R$ operands needed for the CBF calculations in $R$ clock periods.

The complexity for the $\mathrm{N}$-point, radix- $R$ FFT, in number of complex butterflies, is

$$
C_{b}=(N / R) \log _{R} N .
$$

Therefore, the complexity product for rate- $1 / R$ butterflies is

$$
p_{b e} \cdot F_{T}=\log _{R} N \text {. }
$$

Also, it should be noted that this FFT needs a pipeline bit-reverse address calculation for the input to the $0^{\text {th }}$ level memory, and each stage needs two memories, one to act as the input for the following CBF and one to act as the output for the previous CBF, as shown in Figure 1.

Physical Implementation. Specialized digital signal processing devices have been developed that can perform radix 2, 4, and 16 butterflies. An example of this type of device is an ASIC chip introduced by Honeywell ${ }^{1}[5]$. An improved version has been marketed by Sharp [6]. The model of the device that will

\footnotetext{
${ }^{1}$ The mention of commercial products by Honeywell and Sharp does not imply recommendation of the products by the authors or the U.S. government. The use of their chips in a design is based solely on the author's understanding of manufacturers' literature. No evaluation with real hardware units has been conducted.
} 
be considered here is one which performs an operation (e.g., radix 2 butterfly) on a block of data from a source memory to a destination memory. The time required to compute the operation on a data set of length $N$ is $N$ plus $L$ where $L$ is the latency. Further, these devices will have four input/output ports. Operations that will be used to compute the spectral correlation function (SCF) are radix 2, 4, and 16 butterflies, and complex addition and multiplication. Since Fast Fourier Transforms (FFTs) are a major element of computation of the SCF, and the butterfly is the primary operation of FFTs, special purpose chips will be referred to as Butterfly Machines (BFM).

To demonstrate the use of a Butterfly Machine using an architecture that operates in real time, consider the Real-Time FFT Architecture in Figure 1. To compute a $N=1024$ length FFT, three passes are required; one radix 2 pass and two radix 16 passes. This is accomplished by setting the Op Codes to the left most Butterfly Machine to radix 2, and the Op Codes to the other two Butterfly Machines to radix 16. The Data Flow Codes are all set to read data from the left port and write data to the right port. Coefficient data is read from the Coefficient Buffer (not shown in Figure 1). Data is streamed from left to right with one data element entering and leaving the Butterfly Machine for each clock cycle. Output from the last Butterfly Machine is ignored until the third pass. Once the pipeline is full, this architecture produces one FFT every pass. Note that the input to each Butterfly Machine is toggled between Buffer iA and $\mathrm{iB}$ where $\mathrm{i}$ is the level in the pipeline. In general, the number of Butterfly Machines required for data of length $N$ is $\left[\log _{16}(N)\right]$.

An architecture that does not compute in real time, referred to as the One-Chip FFT Architecture, is shown in Figure 2. The algorithm to compute the FFT is identical to the algorithm used for the RealTime FFT Architecture. The only difference is that there is only one Butterfly Machine to perform each radix pass and therefore, data must be passed back and forth between Buffer A and B with the appropriate Op Code set for each pass. Notice that Address Generators for each memory and a Coefficient Buffer are required. These were left out of Figure 1 for brevity.

Use of this architecture to compute a time smoothed SCF will be discussed in the next section. Later, these architectures will be expanded to compute different implementations of the spectral correlation function.

\section{The TDOA Architecture}

The SCA architecture used to analyze signals and to find the TDOA is shown in Figure 3. The key components are

- the SCA which computes a smoothed cyclic periodgram $S_{x_{1 / \Delta f}}^{\alpha}(f)_{\Delta t}$ for all $\alpha \geq 0$ and $f \geq 0$,

- the characterizer and parametizer, which finds signals of interest and computes the auto spectral correlation function of features to be used in TDOA computation, and finds the $\alpha$ of features of interest, a typical $\alpha$ being $\alpha_{0}$
- the CSCA which computes the cross cyclic periodogram, $S_{x y_{A t}}^{\alpha}(f)_{\Delta f_{c}}$, for $\alpha_{0}$ and all $f$,

- and the SPECCOA section which computes the TDOA.

Computation of the SCF. Brown and Loomis have analyzed the theory behind the computation of and the computational complexity of the SCF [7]. The conclusion of this work was that the Strip Spectral Correlation Analyzer (SSCA) was the most computationally efficient method for computing the smoothed SCF over the entire bi-frequency plane. Its functional block diagram is shown in Figure 4. The SSCA is:

- slightly more computationally efficient than the FFT Accumulation Method (FAM),

- significantly more computationally efficient than the Frequency Smoothing Method (FSM) for computing the smoothed SCF over the entire bi-frequency plane,

- produces a time smoothed cyclic periodogram with constant $\Delta f$ over all $f, \alpha$, in contrast to the FAM [7].

Our complexity analysis for the various portions of the TDOA processor will be based on the number of floating point operations, $C_{f p}$, required for each of the principal subcomponents shown in Figure 3. This equals the number of real multiply plus real add operations, as analyzed in [7]. These complexity numbers, $C_{f p}$, and the complexity products, $p_{f p} \cdot F_{T}$, will be used to assess the computational load of the system Finally, we will also analyze $p_{b f m} \cdot F_{T}$ for the computation of the SCF, CSCF, and TDOA using the Butterfly Machine as the pipeline computation unit. Summarizing the results of [7] for the SSCA, we see in Table 1 the complexity summary for the SSCA.

For all of the following realizations, $L P=\Delta t$, $L=N^{\prime} / 4=N /(4 M), P=4 M, \Delta f=1 / N^{\prime}=M / N$, $\Delta \alpha=1 / \Delta t=1 / N$, and $\Delta f \Delta t=N / N^{\prime}=M$, assuming $f_{s}=1$.

We can derive complexity figures in terms of $M$ and $N$ rather than $N^{\prime}, P$, and $L$, resulting in,

$$
C_{f p}=\frac{5 N^{2}}{2 M} \log _{2} N+\frac{3 N^{2}}{M}+20 N \log _{2}(N / M)+16 N
$$

The previous equation, when rewritten in terms of $(\Delta t \Delta f)$ and $\Delta f$ becomes:

$$
\begin{gathered}
C_{f p}=\frac{5 \Delta t \Delta f}{2 \Delta f^{2}} \log _{2}\left[\frac{\Delta t \Delta f}{\Delta f}\right]+\frac{20 \Delta t \Delta f}{\Delta f^{2}} \log _{2}(1 / \Delta f) \\
+\frac{3 \Delta t \Delta f}{\Delta f^{2}}+\frac{16 \Delta \Delta f}{\Delta f}
\end{gathered}
$$

Next, we can compute the hardware complexity product for the real multiply units,

$$
\begin{gathered}
p_{f p} \cdot F_{T}=\left(\frac{5}{2 \Delta f}\right) \log _{2}\left(\frac{\Delta t \Delta f}{\Delta f}\right) \\
+20 \log _{2}(1 / \Delta f)+3(1 / \Delta f)+16 .
\end{gathered}
$$


Table 1: Complexity Summary for SSCA

\begin{tabular}{|c|c|c|c|}
\hline \multicolumn{4}{|c|}{$(P$ required $)$} \\
\hline & Wndw & $N^{\prime}$ FFT & $\begin{array}{c}\text { Down } \\
\text { Convert }\end{array}$ \\
\hline CBF & - & $N^{\prime} / 2 \log _{2} N^{\prime}$ & - \\
\hline Cpx Mpy & - & $N^{\prime} / 2 \log _{2} N^{\prime}$ & $N^{\prime} / 2$ \\
\hline Cpx Add & 二 & $N^{\prime} \log _{2} N^{\prime}$ & - \\
\hline Real Mpy & $N^{\prime}$ & $2 N^{\prime} \log _{2} N^{\prime}$ & $2 N^{\prime}$ \\
\hline Real Add & $\overline{-}$ & $3 N^{\prime} \log _{2} N^{\prime}$ & $N^{\prime}$ \\
\hline BFM Ops & $N^{\prime}$ & $N^{\prime} \log _{16} N^{\prime}$ & $N^{\prime}$ \\
\hline
\end{tabular}

\begin{tabular}{||c|c|c||}
\hline \multicolumn{3}{|c||}{$\left(N^{\prime} / 2\right.$ required $)$} \\
\hline & $\begin{array}{c}\text { Corr. } \\
\text { Mult. }\end{array}$ & $N$ FFT \\
\hline CBF & -- & $N / 2 \log _{2} N$ \\
\hline Cpx Mpy & $N$ & $N / 2 \log _{2} N$ \\
\hline Cpx Add & - & $N \log _{2} N$ \\
\hline Real Mpy & $4 N$ & $2 N \log _{2} N$ \\
\hline Real Add & $2 N$ & $3 N \log _{2} N$ \\
\hline BFM Ops & $N$ & $N \log _{16} N$ \\
\hline
\end{tabular}

If we instead compute as much as possible of the SSCA function using the Butterfly Machine, we use a sequence of various length passes employing the Butterfly Machine set. Each Butterfly Machine pass can be configured to do:

1. $P$ passes, consisting of a windowing real multiply combined with a radix- 2 butterfly, pass length $N^{\prime}$,

2. a $P$ FFTs of length $N^{\prime}$ computed by $\log _{16}\left(N^{\prime}\right)$ Radix-16 Butterfly passes of length $N^{\prime},{ }^{2}$

3. $P$ complex vector multiply passes of length $N$ for down conversion,

4. $N^{\prime} / 2$ complex vector multiply passes of length $N$ for correlation,

5. $N^{\prime} / 2$ length $N$ FFTs, each in turn computed by $\log _{16} N$ length- $N$ Radix-16 butterfly passes. ${ }^{3}$

These operations will result in a total number of Butterfly Machine functional operations, $C_{b f m}$, as given in (17), (18), and (19).

$$
\begin{gathered}
C_{b f m}=P N^{\prime}+P N^{\prime} \log _{16}\left(N^{\prime}\right)+P N^{\prime} \\
+N N^{\prime} / 2+\left(N N^{\prime} / 2\right) \log _{16}(N)
\end{gathered}
$$

Written in terms of $N / M=N^{\prime}$ and $4 M=P$,

$C_{b f m}=\frac{N^{2}}{M} \log _{16}(N)+\frac{N^{2}}{M}+4 N \log _{16}\left(\frac{N}{M}\right)+8 N$

Finally, using the previously given relations, $C_{b f \mathrm{~m}}$, as a function of $\Delta t \Delta f$ and $\Delta f$ is

$$
C_{b f m}=\frac{\Delta t \Delta f}{\Delta f^{2}} \log _{16}\left(\frac{\Delta t \Delta f}{\Delta f}\right)+\frac{\Delta t \Delta f}{\Delta f^{2}}
$$

\footnotetext{
${ }^{2}$ We assume that $N^{\prime} / 2$ is 16 raised to an integer power.

${ }^{3}$ We assume that $N$ is 16 raised to an integer power.
}

$$
+\frac{4 \Delta t \Delta f}{\Delta f} \log _{16}\left(\frac{1}{\Delta f}\right)+\frac{8 \Delta t \Delta f}{\Delta f} .
$$

Next, we can compute the complexity product, $p_{b f m} \cdot F_{T}$, for a totally Butterfly Machine realization of the SSCA as

$$
\begin{gathered}
p_{b f m} \cdot F_{T}=(1 / \Delta f) \log _{16}\left(\frac{\Delta t \Delta f}{\Delta f}\right) \\
+(1 / \Delta f)+4 \log _{16}\left(\frac{1}{2 \Delta f}\right)+8
\end{gathered}
$$

Equation (20) tells us how to utilize Butterfly Machine to compute the SCF using the SSCA for any desired factor of real time.

Architectural Tradeoffs. An architecture for computing both time and frequency smoothed spectral correlation functions is shown in Figure 5 . The One-Chip Architecture computes spectral correlation functions with minimal hardware. It is an enhanced version of the One-Chip FFT Architecture shown in Figure 2 by providing more variety in data flow, and by adding another memory referred to as the Auxiliary Buffer. The Auxiliary Buffer is used to store intermediate values.

To illustrate the computation of the SSCA using the One-Chip Architecture, consider Table 1 and Figure 4. First $P$ windowed FFTs of length $N^{\prime}$ must be computed. For each $N^{\prime}$ FFT, this is accomplished by using first a windowed 2 or 4 radix pass followed by 2,4 , or 16 radix passes based on the value of $N^{\prime}$ to complete the $N^{\prime}$ FFT. Buffers A and B are used with the resulting FFT data stored in one of these buffers. Weighting factors and windowing coefficients are stored in the Coefficient Buffer. This is followed by $P$ complex multiply passes to down convert the data. The correlation multiplies are completed using another complex multiply pass with results sent to the Auxiliary Buffer. A total of $N^{\prime} / 2$ FFTs of length $N$ are then computed with data passed between Buffer $B$ and the Auxiliary Buffer. The last pass is sent to the Output Port.

A real-time architecture for computing SSCA is shown in Figure 6 . Referring again to Figure 3 and Table 1, the Front End in Figure 6 is responsible for computing the $P$ FFTs of length $N^{\prime}$ as well as the down conversions. A typical decimation factor is $N^{\prime} / 4$, therefore a minimum of four parallel pipelines are necessary to compute these FFTs in real time. At the end of each of these pipelines is another Butterfly Machine for computing the complex multiply for down-conversion.

The down-converted data is passed to the Back End Channels. Each Back-End channel must be sized for length $N$ data. Each channel has one Butterfly Machine for the correlation multiply pass followed by a pipelined FFT of length $N$. For real input data, there are $N^{\prime} / 2$ Back-End Channels for this architecture to perform in real time.

The One-Chip Architecture and Real-Time Architecture represent the extremes of possible architectures with the One-Chip Architecture representing the slowest but least expensive architecture and the Real Time Architecture representing the fastest and 
Table 2: Cross FSM Complexity

\begin{tabular}{||c|c||c|c||}
\hline \multicolumn{2}{||c||}{ (2 required) } & \multicolumn{2}{c||}{$N / 2 M$ required } \\
\hline & FFT & Correlator & Summer \\
\hline CBF & $(N / 2) \log _{2} N$ & - & - \\
\hline Cpx Mpy & $(N / 2) \log _{2} N$ & $M$ & - \\
\hline Cpx Add & $N \log _{2} N$ & - & $M$ \\
\hline Real Mpy & $2 N \log _{2} N$ & $4 M$ & - \\
\hline Real Add & $3 N \log _{2} N$ & $2 M$ & $2 M$ \\
\hline BFM Ops & $N \log _{16} N$ & $M$ & $M$ \\
\hline
\end{tabular}

most expensive. There are architectural options between these extremes. Equation (17) can be partitioned as:

$$
\begin{aligned}
& C_{B F E}=P N^{\prime}+P N^{\prime} \log _{16}\left(N^{\prime}\right)+P N^{\prime} \\
& C_{B B E}=N N^{\prime} / 2+\left(N N^{\prime} / 2\right) \log _{16}(N)
\end{aligned}
$$

representing the number of computations required by the Front End and Back End, respectively. Equations (21) and (22) can be used to allocate available Butterfly Machines to the Front and Back Ends when an intermediate architecture is desired.

Computation of the CSCA. Once the features of the SOI(s) are identified, it is then the task of CSCA to compute the value of the function $S_{x y_{\Delta t}}^{\alpha}(f)_{\Delta f_{c}}$ for a specific value of $\alpha=\alpha_{0}$, and for an increment in $f$, $\Delta f_{c}$, which must satisfy the following:

$$
\Delta f_{c}<1 / d
$$

where $d$ is the delay difference in the signal between $x$ and $y[3]$.

Here, the FAM and SSCA architectures lose their advantage, as seen in the complexity analysis for a single $\alpha$. This is caused by the fact that the FFT based time smoothing architectures achieve efficiency by computing a large number ( $\mathrm{P}$ or $\mathrm{N})$ values of $S_{x y_{1 / \Delta f}}^{\alpha}(f)_{\Delta t}$ at once.

Figure 7 , illustrates the functionality for computing $S_{x y_{\Delta t}}^{\alpha_{0}}(f)_{\Delta f_{c}}$.

A complexity analysis is conducted on Figure 7 the CFSM architecture and the results tabulated in Table 2.

Table 2 summarizes the complexity analysis in floating point operations for the major sections of the FSM realization for one value of $\alpha$.

$$
\begin{gathered}
C_{f p}=4 N+10 N \log _{2} N \\
C_{f p}=\frac{4\left(\Delta t \Delta f_{c}\right)}{\left(\Delta f_{c}\right)}+\frac{10\left(\Delta t \Delta f_{c}\right)}{\Delta f_{c}} \log _{2}\left[\frac{\Delta t \Delta f_{c}}{\Delta f_{c}}\right] .
\end{gathered}
$$

The complexity product for floating point operations is

$$
p_{f p} \cdot F_{T}=4+10 \log _{2}\left(\frac{\Delta t \Delta f_{c}}{\Delta f_{c}}\right) .
$$

The complexity in Butterfly operations, $C_{b f m}$, for the cross FSM, developed from the last row of Table 2 is

$$
C_{b f m}=2 N \log _{16} N+N,
$$

or

$$
C_{b f m}=\frac{2 \Delta t \Delta f_{c}}{\Delta f_{c}} \log _{16}\left(\frac{\Delta t \Delta f_{c}}{\Delta f_{c}}\right)+\frac{\Delta t \Delta f_{c}}{\Delta f_{c}}
$$

The corresponding complex product is

$$
p_{b f m} F_{T}=2 \log _{16}\left(\frac{\Delta t \Delta f_{c}}{\Delta f_{c}}\right)+1
$$

Roberts has suggested a more efficient method than the CFSM for computing single- $\alpha$ cross spectra called the one bit spectral correlation analyzer (OBSCA). That algorithm replaces the correlation complex multiplication operation by a sign change (multiplication by \pm 1 ) [9]. OBSCA results in significant reduction in the number of real multiplies required over CFSM, but no improvement is seen in the number of real additions. Furthermore, there is no advantage to using OBSCA with a pipeline processor such as the Butterfly Machine. More detailed analysis of the benefits of OBSCA is carried out in [10]. While it is not treated further in this paper, the reader is urged to carefully consider its use in the computation of $S_{x y_{\Delta t}}^{\alpha}(f)_{\Delta f_{c}}$ for a single $\alpha$.

TDOA Computation. The final operation to be preformed by the TDOA processor of Figure 3 is the computation of the TDOA itself from $S_{x y_{1 / \Delta f}}^{\alpha_{0}}(f)_{\Delta t}$ and $S_{x y_{\Delta t}}^{\alpha_{0}}(f)_{\Delta f_{c}}$. The algorithm chosen for this paper is SPECCOA, not only because of the slight improved performance cited earlier, but also because pipeline multiply is substantially less complex than pipeline division required by SPECCOR. The inverse FT operation in the Equation (10) is implemented by an FFT over a discrete set of products for values of $f$ separated by the increment $\Delta f_{c}$, which may be less than the $\Delta f$ used for computing $S_{x y_{\Delta}}^{\alpha}(f)_{\Delta t}$. If that is the case, intermediate values of $S_{x 1 / \Delta f}^{\alpha_{0}}(f)_{\Delta t}$ can be interpolated. The inverse FFT in (10) will be computed over $N_{c}^{\prime}$ points where $N^{\prime}=N / M_{\mathrm{c}}$ and $\Delta f_{c}=1 / N_{c}^{\prime}$.

The operations required by SPECCOA are tabulated in Table 3

From Table 3 , the number of real multiplies required and the complexity product for real multiplies can be developed. These are given in equations (30) and (31).

$$
\begin{aligned}
C_{f p} & =2 N_{c}^{\prime} \log _{2} N_{c}^{\prime}+4 N_{c}^{\prime} \\
& =\frac{2}{\Delta f} \log _{2}\left(1 / \Delta f_{c}\right)+\frac{4}{\Delta f_{c}} \\
p_{f p} F_{T} & =\frac{2}{\Delta f_{c} \Delta t} \log _{2}\left(1 / \Delta f_{c}\right)+\frac{4}{\Delta f_{c} \Delta t}
\end{aligned}
$$

The complexity analysis for Butterfly chips yields equations (32) and (33).

$$
\begin{aligned}
C_{b f m} & =N_{c}^{\prime} \log _{16} N_{c}^{\prime}+2 N_{c}^{\prime} \\
& =\left(1 / \Delta f_{c}\right) \log _{16}\left(1 / \Delta f_{c}\right)+\left(2 / \Delta f_{c}\right)(31)
\end{aligned}
$$


$p_{b f m} F_{T}=\frac{1}{\Delta f_{c} \Delta t} \log _{16}\left(1 / \Delta f_{c}\right)+\left(\frac{2}{\Delta f_{c} \Delta t}\right)$

Table 3: Complexity Summary for SPECCOA

\begin{tabular}{||c|c||c||}
\hline \multicolumn{2}{||c||}{ Inverse FFT } & Product \\
\hline 1 required & FFT & 2 required \\
\hline & Correlator \\
\hline CBF & $N_{c}^{\prime} / 2 \log _{2} N_{c}^{\prime}$ & - \\
\hline Cpx Mpy & $N_{c}^{\prime} / 2 \log _{2} N_{c}^{\prime}$ & $N_{c}^{\prime}(1)$ \\
\hline Cpx Add & $N_{c}^{\prime} \log _{2} N_{c}^{\prime}$ & - \\
\hline Real Mpy & $2 N_{c}^{\prime} \log _{2} N_{c}^{\prime}$ & $4 N_{c}^{\prime}$ \\
\hline Real Add & $3 N_{c}^{\prime} \log _{2} N_{c}^{\prime}$ & $2 N_{c}^{\prime}$ \\
\hline BFM Ops & $N_{c}^{\prime} \log _{16} N_{c}^{\prime}$ & $N_{c}^{\prime}$ \\
\hline
\end{tabular}

Note: (1) Maximum value.

\section{Summary and Conclusions}

We have shown the architecture of the TDOA computing SCA for two independently received signals $x$ and $y$. The overall architecture was presented in Figure 3 .

The real multiplier complexity products for each of the major components were presented in equations (16), (23), and (29).

$$
\begin{gathered}
\mathrm{SSCA}-S_{x 1 / \Delta f}^{\alpha}(f)_{\Delta t} \\
p_{f p} \cdot F_{T}=(5 / 2 \Delta f) \log _{2}\left(\frac{\Delta t \Delta f}{\Delta f}\right) \\
+20 \log _{2}(1 / \Delta f)+3(1 / \Delta f)+16 \\
\mathrm{CFSM}-S_{x y_{\Delta t}}^{\alpha_{0}}(f)_{\Delta f_{c}} \\
p_{f p} \cdot F_{T}=4+10 \log _{2}\left(\frac{\Delta t \Delta f_{c}}{\Delta f_{c}}\right)+2 \\
\mathrm{SPECCOA}-\mathrm{TDOA} \\
p_{f p} \cdot F_{T}=\frac{2}{\Delta f_{c} \Delta t} \log _{2}\left(1 / \Delta f_{c}\right)+\frac{4}{\Delta f_{c} \Delta t}
\end{gathered}
$$

It is easy to see from the above that the computation of the TDOA for some factor of real time, $F_{T}$, requires the most hardware units, $p_{f p}$, to be devoted to the SCF for all $f$ and $\alpha$ followed by the cross spectrum, CFSM, with the least hardware required by the computation of the SPECCOA product and the inverse FFT.

Similar comparisions for the use of pipeline vector processor chips such as the Butterfly Machine yield equations $(20)$ and $(26)$.

$$
\begin{aligned}
& \operatorname{SSCA}-S_{x_{1 / \Delta f}}^{\alpha}(f)_{\Delta t} \\
& p_{b f m} \cdot F_{T}=(1 / \Delta f) \log _{16}\left(\frac{\Delta t \Delta f}{\Delta f}\right) \\
& \quad+(1 / \Delta f)+4 \log _{16}\left(\frac{1}{2 \Delta f}\right)+8
\end{aligned}
$$

$$
\begin{gathered}
\text { CSFM }-S_{x y_{\Delta t}}^{\alpha_{0}}(f)_{\Delta f_{c}} \\
p_{b f m} \cdot F_{T}=2 \log _{16}\left(\frac{\Delta t \Delta f_{c}}{\Delta f_{c}}\right)+1 \\
\text { SPECCOA }- \text { TDOA } \\
p_{b f m} \cdot F_{T}=\frac{1}{\Delta f_{c} \Delta t} \log _{16}\left(1 / \Delta f_{c}\right)+\left(\frac{1}{\Delta f_{c} \Delta t}\right)
\end{gathered}
$$

In this paper we have shown the overall architecture for finding appropriate cyclic features of a signal and the related value of $\alpha_{0}$, the computation of the cross spectrum for that $\alpha_{0}$ and the calculation of the TDOA using SPECCOA.

We concluded that SSCA was best for $S_{x}^{\alpha}(f)$, CSFM or perhaps OBSCA was best for $S_{x y}^{\alpha_{0}}(f)$ and that SPECCOA was the method to be used to calculate TDOA

Finally, we also tabulated hardware requirements if a commercial pipeline processor chip-set were to be used. From the derived equations, chip requirements and allocations to tasks can be computed for any desired factor of real time.

\section{References}

[1] W. A. Gardner, "Exploitation of spectral redundancy in cyclostationary signals," IEEE Signal Processing Magazine, Vol. 8, No. 2, pp. 38-49 April 1991.

[2] W. A. Gardner and C. K. Chen, "Signal-selective time-difference-of-arrival estimation for passive location of manmade signal sources in highly corruptive environments. Part I: Theory and method," IEEE Trans. on Signal Processing, Vol. SP-40, No. 5, pp. 1168-1184, May 1992.

[3] C. K. Chen and W. A. Gardner, "Signal-selective time-difference-of-arrival estimation for passive location of manmade signal sources in highly corruptive environments. Part II: Algorithms and performance," IEEE Trans. on Signal Processing Vol. SP-40, No. 5, pp. 1185-1197, May 1992.

[4] A. V. Oppenheim and R. W. Schafer, Digital Signal Processing, Englewood Cliffs, NJ: Prentice Hall, 1975.

[5] Honeywell, Inc., "Digital Signal Processing Chip Set," Publication No. DSP-6008/489, Rev. A $3 / 88$, Colorado Springs, CO.

[6] Sharp, Inc., "LH9124 Users Manual," Los Angeles, CA, 1991.

[7] W. A. Brown and H. H. Loomis, Jr., "Digital implementations of spectral correlation analyzers," IEEE Trans. on Signal Processing, Vol. 41, No. 2, pp. 703-720, February 1993.

[8] R. S. Roberts, W. A. Brown, and H. H. Loomis, Jr., "Computationally efficient algorithms for cyclic spectral analysis," IEEE Signal Processing Magazine, Vol. 8, No. 2, pp. 38-49, April 1991. 
[9] R. S. Roberts, "Architectures for digital cyclic spectral analysis," Ph.D. dissertation, University of California, Davis, September 1989.
[10] G. A. Hutcheson, "Implementation of the One Bit Spectral Correlation Algorithm," M.S. Thesis, Naval Postgraduate School, Monterey, CA, June 1992.

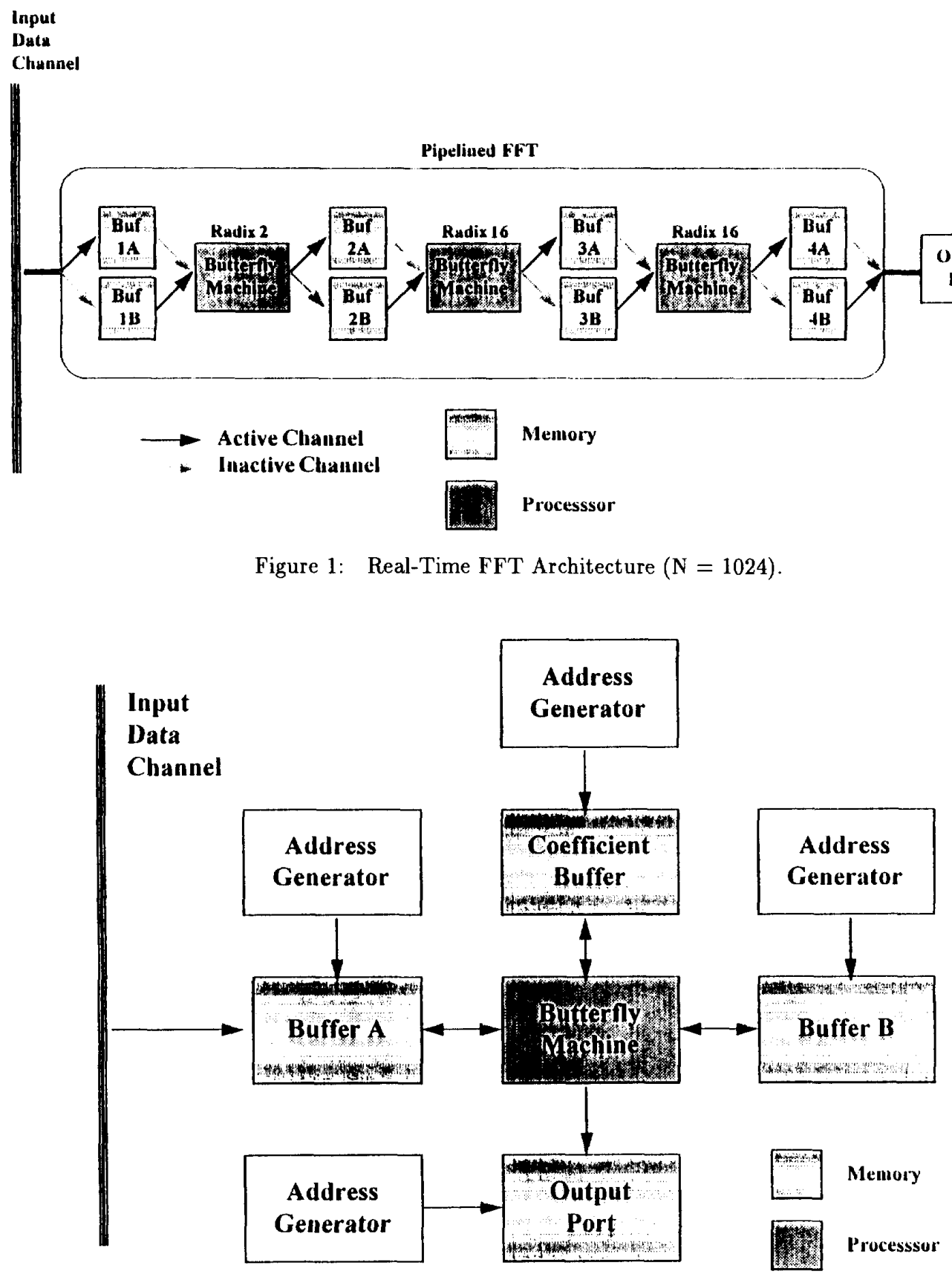

Figure 2: One-Chip FFT Architecture. 


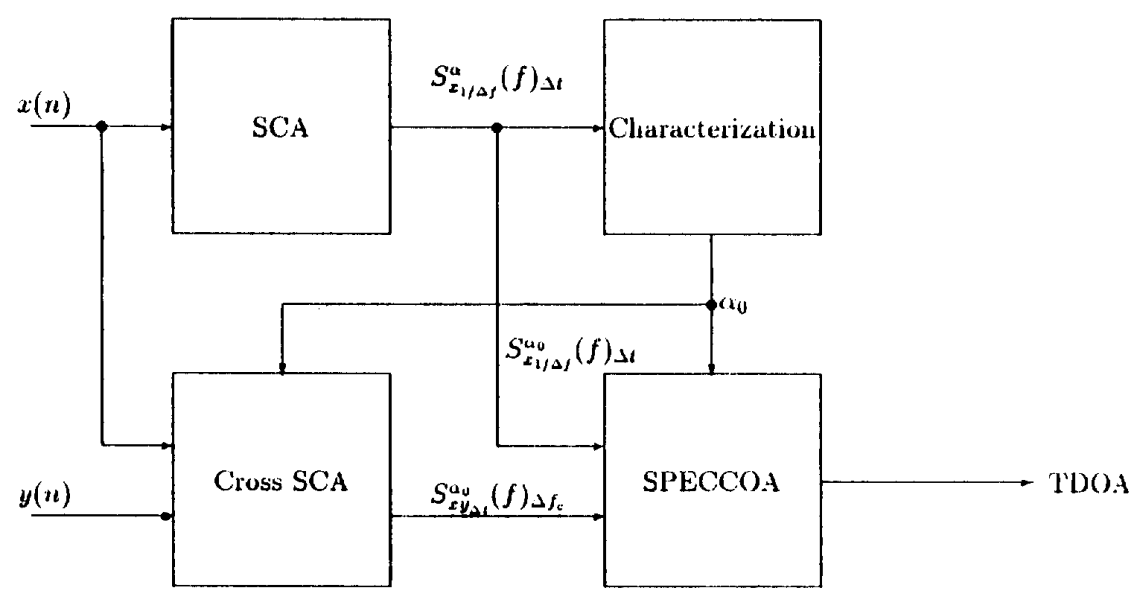

Figure 3: Functional Block Diagram - TDOA Processor.

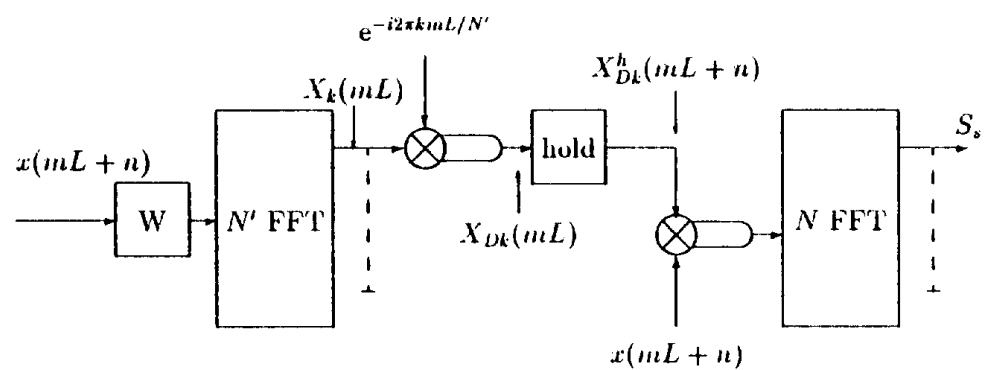

Figure 4: Strip Spectral-Correlation-Analyzer Architecture, $S_{x y_{1 / \Delta f}}^{\alpha}(f)_{\Delta t}$.

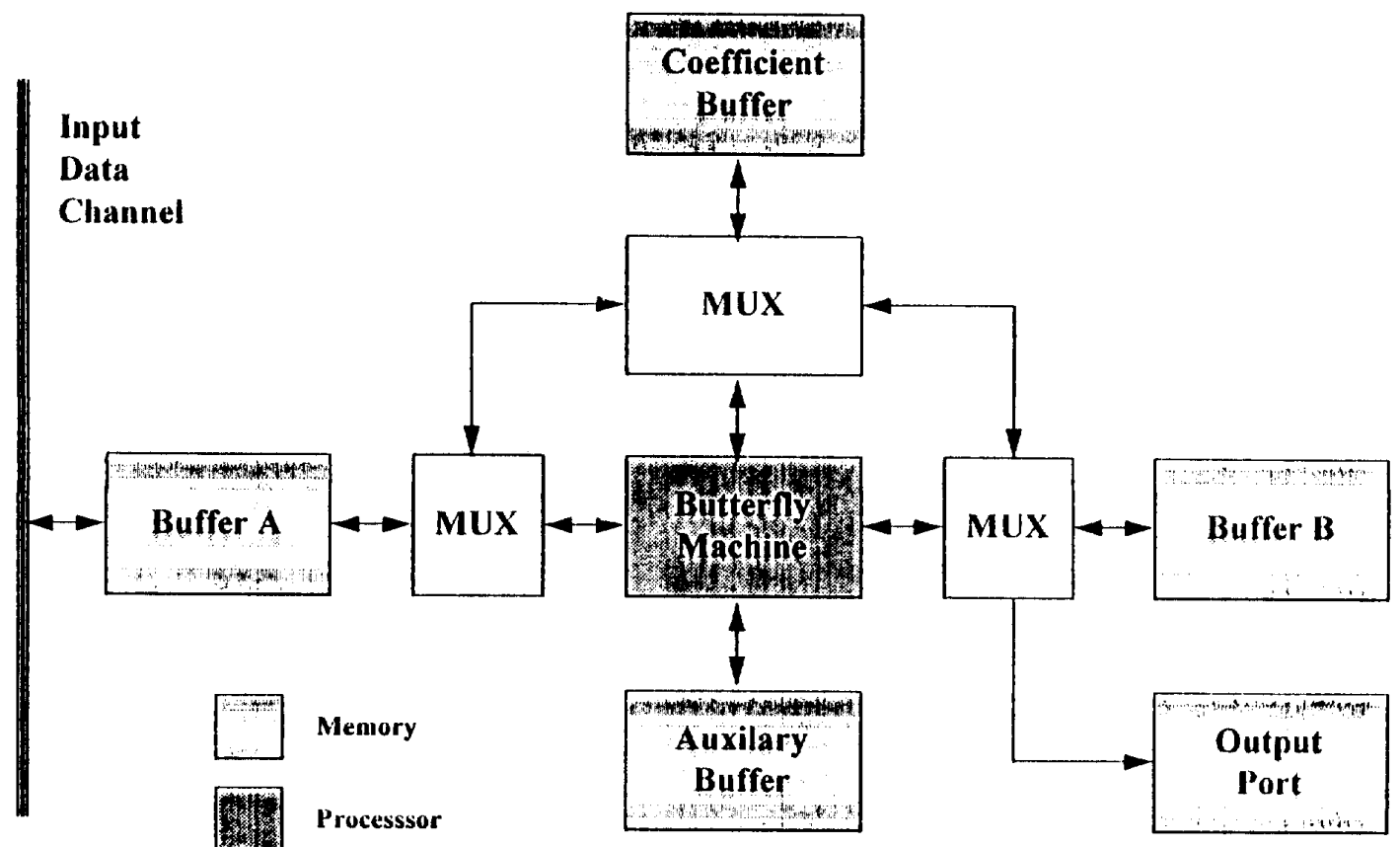

Figure 5: One-Chip Architecture. 


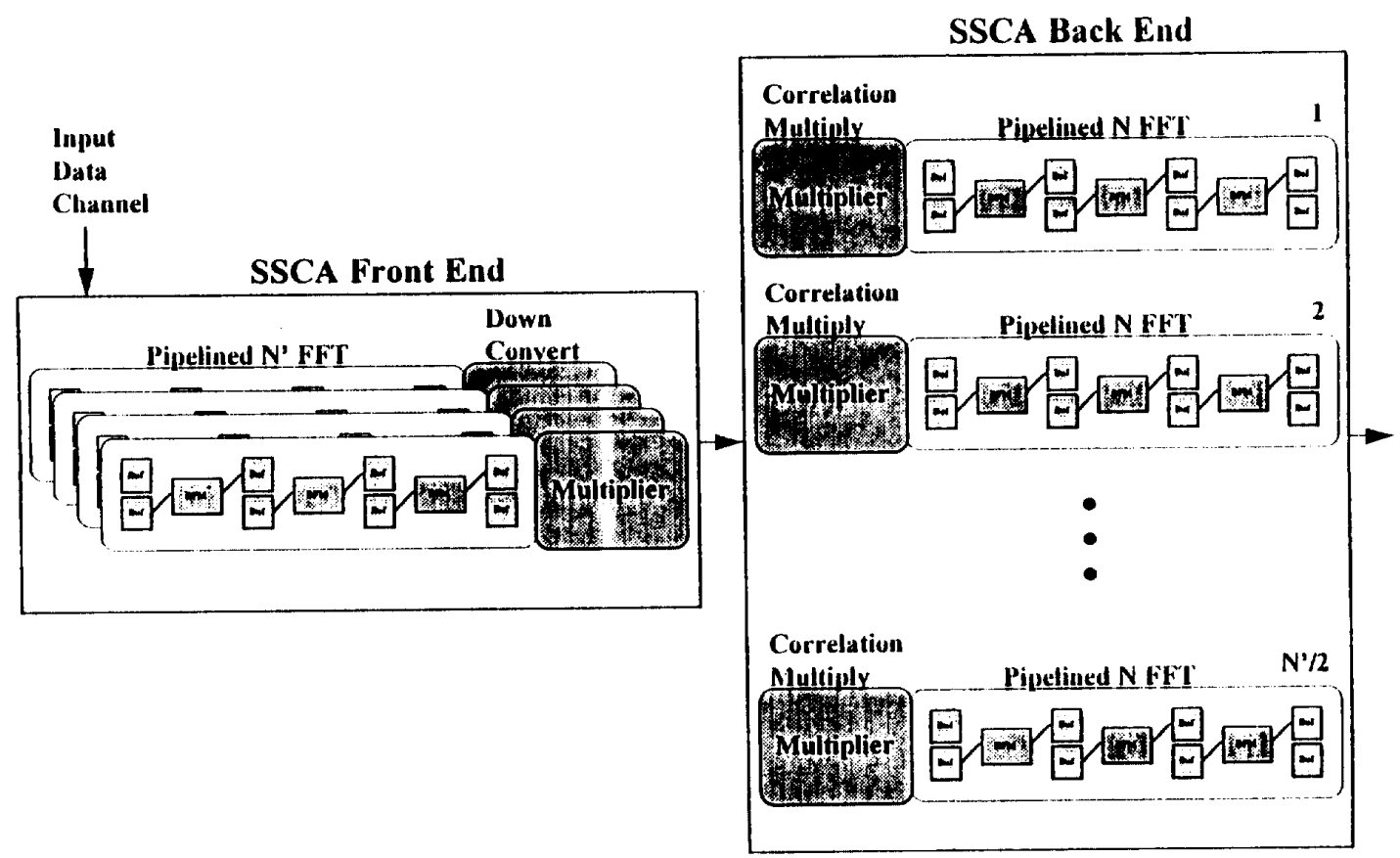

Figure 6: Real-Time Architecture.

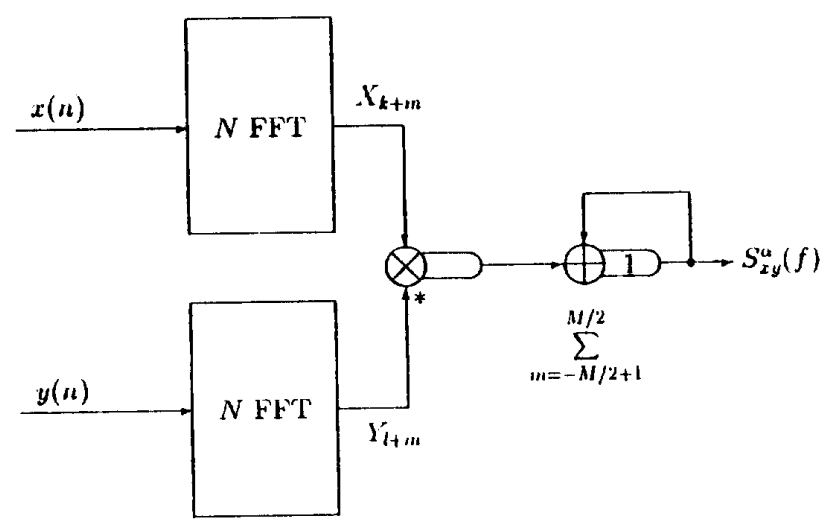

Figure 7: Frequency-Smoothing-Method Architecture - Cross Spectrum, $S_{x y_{\Delta t}}^{\alpha_{0}}(f)_{\Delta f_{c}}$. 